\title{
Erratum - Maternal Mortality in Brazil: Proposals and Strategies for its Reduction
}

\section{Mortalidade materna no Brasil: propostas e estratégias para sua redução}

Rodolfo Carvalho Pacagnella ${ }^{1}$ Marcos Nakamura-Pereira ${ }^{2}$ Flavia Gomes-Sponholz ${ }^{3}$

Regina Amélia Lopes Pessoa de Aguiar ${ }^{4}$ Gláucia Virginia de Queiroz Lins Guerra ${ }^{5}$ Carmen Simone Grilo Diniz ${ }^{6}$ Brenno Belazi Nery de Souza Campos ${ }^{7}$ Eliana Martorano Amaral ${ }^{1,7}$ Olímpio Barbosa de Moraes Filho ${ }^{8}$

\footnotetext{
1 Universidade Estadual de Campinas, Campinas, SP, Brazil

2 Instituto Nacional de Saúde da Mulher, da Criança e do Adolescente

Fernandes Figueira, Rio de Janeiro, RJ, Brazil

${ }^{3}$ School of Nursing, Universidade de São Paulo, Ribeirão Preto, SP, Brazil

${ }^{4}$ Universidade Federal de Minas Gerais, Belo Horizonte, MG, Brazil

5 Instituto de Medicina Integral Professor Fernando Figueira, Recife, PE, Brazil

6 Universidade de São Paulo, São Paulo, SP, Brazil

${ }^{7}$ Faculdade de Medicina São Leopoldo Mandic, Campinas, SP, Brazil

${ }^{8}$ Universidade de Pernambuco, Recife, PE, Brazil
}

Rev Bras Ginecol Obstet 2019;41:65.

\section{ERRATUM}

Rio de Janeiro, December 11, 2018

Dear readers,

In the E-Poster Maternal Mortality in Brazil: Proposals and Strategies for its Reduction (Rev Bras Ginecol Obstet 2018;40.09: 501-506_4010ed. DOI: 10.1055/s-0038-1672181.), published online in Rev Bras Ginecol Obstet in September 2018, where it reads:

Brenno Belazi Nery de Souza Campos ${ }^{7}$ Eliana Martorano Amaral ${ }^{1,7}$

It should read:

Brenno Belazi Nery de Souza Campos ${ }^{1,7}$ Eliana Martorano Amaral ${ }^{1}$ 